\title{
ANTIBIOTIC SENSITIVITY TESTS: A RAPID METHOD SUITABLE FOR MULTIPLE CULTURES
}

\author{
BY \\ J. G. P. HUTCHISON \\ From the Department of Bacteriology, Royal Victoria Infirmary, Newcastle-upon-Tyne
}

(RECEIVED FOR PUBLICATION FEBRUARY 24, 1954)

The apparatus described was designed to deal with a large batch of sensitivity tests with rapidity and accuracy. The method is suitable for routine laboratories where many such tests are demanded.

\section{Method}

An illuminated grid system is used for the orientation of cultures and of blood agar plates containing suitable antibiotic concentrations. A small drop of a broth culture is seeded on to each plate in a position defined on a duplicated paper facsimile of the grid.

Details of the Grid Construction.-Figs. 1-4 show the general design of the apparatus.

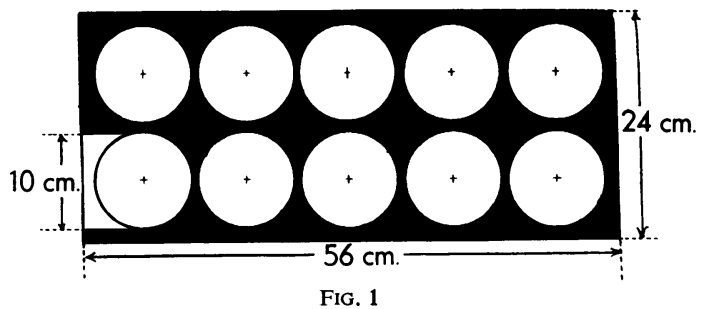

A sheet of "perspex" $24 \times 56 \times 0.3 \mathrm{~cm}$. forms the main table beneath which 10 opaque grids are stuck. The 10 grids of black $x$-ray backing paper are cut simultaneously round an inverted petri-dish lid $(10 \mathrm{~cm}$. with a sharp knife. A circular template of white paper (10 cm. diameter) is ruled into $1.0 \mathrm{~cm}$. squares (Fig. 2 lower half) and those for subsequent perforation marked (Fig. 2 upper half); all discs are then stapled together. With a gentle screwing motion, a very sharp cork borer, giving a hole ó $\mathrm{mm}$. in diameter, is driven through the disc pad in the centre of each marked square. On completion the staples are removed and the 10 identical black grids stuck to the " perspex" table. A very thin glue of perspex shavings in chloroform may be used, the grid being immersed in the glue and quickly transferred to its exact position; owing to rapid setting, no change is possible once the grid is in place. Subsequently transparent areas between the grids are coated with dead-black paint (Fig. 1 in black).

The completed table is mounted in a frame which pulls out from beneath the laboratory bench like a drawer (Fig. 3).
Illumination.-A 100 -watt lamp with a metal reflector is fixed to the underside of the bench not less than $2 \mathrm{ft}$. from the grid system in a working position. An aluminium reflector $(56 \times 30 \mathrm{~cm}$.) attached to the draw-out frame and inclined at about $45^{\circ}$ changes the direction of light from the horizontal so that it shines vertically upward through each grid. The reflector is mounted on hinges and can be folded up against the underside of the grid table when not in use.

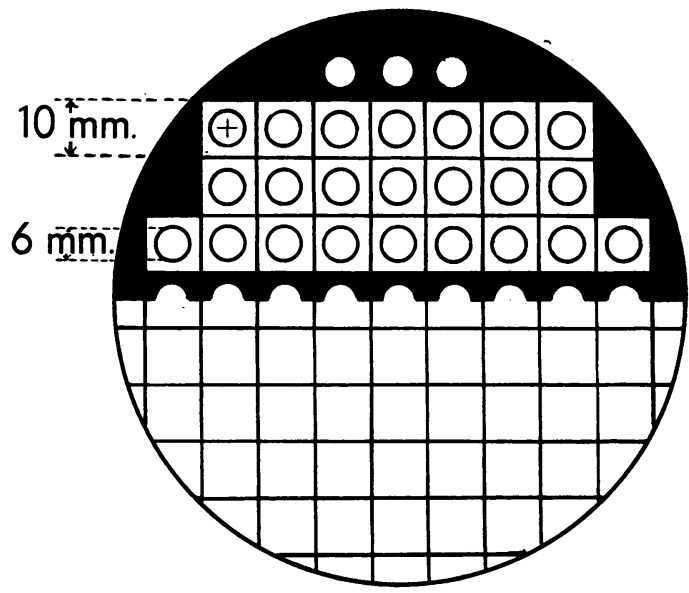

Fig. 2

Pipettes.-Pasteur pipettes are drawn from $5 \mathrm{~mm}$. glass tubing into fine pliable points. The exact relationship between end-bore and flexibility is a matter of experience, but they should allow about $0.05 \mathrm{ml}$. of broth to be drawn up in a matter of seconds: if too fine filling is slow, if too rigid inoculation of the plates is made difficult, since they may stick in the agar and become choked or deliver too large a drop.

Sterile pipettes are stored in aluminium cans. After use each is flushed with distilled water, boiled, and resterilized at $160^{\circ} \mathrm{C}$.

Identification.-For identification, culture numbets are written down on forms (prepared on a duplicator) bearing a squared facsimile of the grid (Fig. 2 lower half).

Antibiotics.-Antibiot ${ }_{\text {Ics }}$ are incorporated at suitable concentrations in a blood agar medium. For routine 


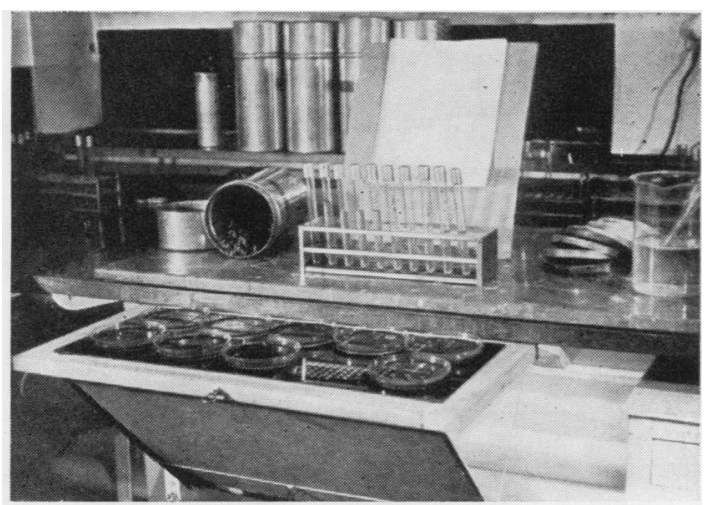

Fig. 3.-A view of the apparatus sho wing the general layout of the grid table and reflector together with accessories.

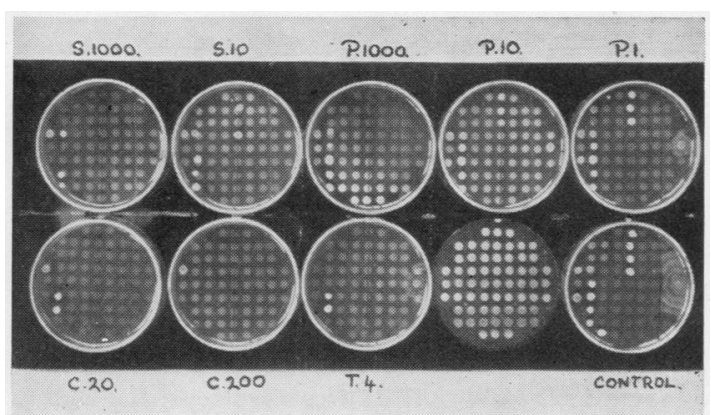

FIG. 4.-The grid table with plates laid out as for inoculation. One plate has been removed to show the grid; the remainder have been inoculated with a few cultures and incubated to show growth. The plates in this case contain: penicillin 1.0,10, and 1,000 units per ml.; streptomycin 10 and $1,000 \mu \mathrm{g}$. per ml.; chloromycetin 20 and $200 \mu \mathrm{g}$. per $\mathrm{ml}$.; and terramycin $4 \mu \mathrm{g}$. per $\mathrm{ml}$. purposes penicillin (at three levels), streptomycin, chloromycetin, and terramycin (two levels each) are used, a fresh batch of plates $(10 \mathrm{~cm}$. diameter) being poured weekly.

Test-tubes.-Test-tubes with "oxoid" aluminium caps containing broth or peptone water are seeded and incubated for four hours at $37^{\circ}$ for inoculation.

\section{Procedure}

Each petri dish is first marked across its diameter with a line (in black) bearing a cross at one end. This diameter, when a plate is in the correct position, falls north-south down the centre of the grid over which it is placed, with the cross to north. The plates in position, a pipette is charged with about $0.05 \mathrm{ml}$. of the first broth culture and a 3-6 mm. spot touched off on to each agar surface in turn. Subsequent cultures follow in the same manner so that in any one series of plates a given specimen occupies an identical position on each. It is found easier to inoculate successive cultures in a north to south direction. After drying the plates are incubated.

Reading is done most easily by taking one plate at a time and noting all reactions in a suitable book. Since it is the crux of the method that all cultures and their corresponding numbersmust follow an invariable sequence the duplicate form need rarely be consulted.

Speed of performance may be judged from the fact that after some practice the inoculation time of 10 plates for 60 cultures is about 30 minutes and reading time 20 minutes.

The method has been in routine use in this department for a year and has proved satisfactory.

My thanks are due to Dr. C. A. Green of this department for valuable help in connexion with this paper. 